\title{
TEMA 10-2016: Manejo de masas anexiales
}

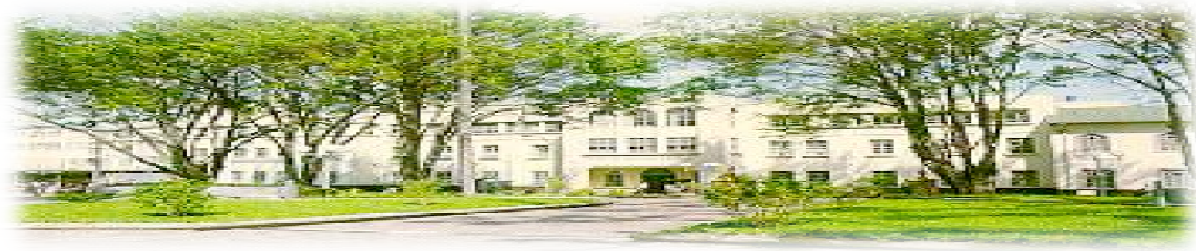

Hospital San Juan de Dios, San José, Costa Rica. Fundado en 1845
Recibido:
$01 / 12 / 2015$
Aceptado:
$/ 01 / 2016$

Melissa Mora Delgado ${ }^{1}$ Roberto del Valle Solano ${ }^{2}$

\footnotetext{
${ }^{1}$ Médico General, Hospital Metropolitano, correo electrónico: mmora0489@gmail.com

${ }^{2}$ Especialista en Ginecología y Obstetricia,Servicio de Ginecología y Obstetricia Hospital San Vicente de Paul. Heredia
}

\section{RESUMEN}

El manejo de una masa anexial se enfoca principalmente en descartar el riesgo de malignidad existente. $\mathrm{Y}$ así definir el tratamiento. El diagnóstico se basa en hallazgos ultrasonográficos, así como factores de riesgo y edad de la paciente. Una masa anexial de bajo riesgo de malignidad se aborda mediante observación con US control o mediante cirugía laparoscópica, mientras una masa con moderado o alto riesgo de malignidad debe abordarse por vía quirúrgica, vía laparotomía en la mayoría de los casos.

\section{PALABRAS CLAVE}

Masa anexial, cáncer de ovario, quistes de ovario, ultrasonografia, quiste ovario, ca-125, HE4.

\section{ABREVIATURAS}

US: Ultrasonido

EPI: Enfermedad pélvica Inflamatoria

HE4:Human Epididymis Protein 4

FDA: Food and Drug Administration
RMI: Resonancia Megnética

ACO: Anticonceptivo Oral

\section{ABSTRACT}

The management of an adnexal mass focuses primarily in ruling out the risk of malignancy and defining treatment. The diagnosis is based on ultrasound findings, as well as risk factors and age of the patient. An adnexal mass at low risk of malignancy will be managed conservative by observation or by laparoscopic surgery, while a mass at moderate or high risk of malignancy should be addressed surgically, via laparotomy in the majority of cases.

\section{KEY WORDS}

Masa anexial, cáncer de ovario, quistes de ovario, ultrasonografía, quiste ovario, ca-125, HE4.

\section{INTRODUCCIÓN}

La nueva práctica en el uso de Ultrasonido de rutina en las consultas ginecológicas,así como en las con- 
sultas de medicina general preventiva, determina un aumento en la frecuencia de detección de masas anexiales. Esto conlleva una importancia en el conocimiento sobre el manejo de dichos hallazgos, la mayoría incidentales.

El mayor objetivo en el manejo de masas anexiales es la exclusión de malignidad. Esto basándose en dos aspectos primordiales como lo es la edad de la paciente y características propias de la masa.

El cáncer de ovario representa la causa principal de mortalidad por malignidades ginecológicas, con una sobrevivencia a 5 años del $40 \%$, especialmente relacionada con la poca capacidad de detección en etapas tempranas.

No existe método de screening efectivo, que haya demostrado significancia clínica para detección de cáncer de ovario. ${ }^{(1,8)}$

\section{DISCUSIÓN}

\section{Definición}

Masa anexial es aquella masa encontrada ya sea en las trompas de Falopio, el ligamento redondo, los ovarios y las estructuras internas del ligamento ancho. Así como órganos adyacentes que pueden estar involucrados. Sin embargo, el término masa anexial es inespecífico en la práctica clínica y frecuentemente se asocia con tumor o quiste ovárico, ello debido a que la patología tubárica es poco prevalente..$^{(1,6)}$

\section{Incidencia}

Las masas anexiales son una patología común tanto en mujeres premenopáusicas, como postmenopáusicas. La gran mayoría corresponden a entidades benignas, siendo un bajo porcentaje cáncer de ovario.Aparecen sobre todo en edad reproductiva (20-45 años); las causas más frecuentes en esta época son las de origen funcional. En prepúberes, perimenopáusicas y postmenopáusicas deben ser objeto de estudio inmediato, por el aumento del riesgo de malignidad. Aproximadamente un $10 \%$ de las mujeres, recibirán tratamiento quirúrgico debido a una masa anexial en sus vidas y se estima que en Estados Unidos 60.000 cirugías al ano se practican debido a esta causa. ${ }^{(1,4)}$

\section{Diagnóstico}

El diagnóstico diferencial de una masa anexial es fundamental para así definir la necesidad de tratamiento, ya sea quirúrgico o conservador.

En el diagnóstico se deben diferenciar causas no ginecológicas y ginecológicas. Entre las ginecológicas se presentan causas funcionales, inflamatorias y tumorales (benignas o malignas). (cuadro $\mathrm{I}) .^{(1,8)}$

Cuadro I.Principales causas de masas anexiales clasificadas según edad de aparición y etiología.

\begin{tabular}{|l|l|l|l|}
\hline EDAD & FUNCIONALES & INFLAMATORIAS & TUMORAL \\
\hline PREMENARCA & $\begin{array}{l}\text { Quiste Funcional } \\
\text { (folicular, } \\
\text { hemorrágico, } \\
\text { cuerpo lúteo) }\end{array}$ & $\begin{array}{l}\text { EPI } \\
\text { Endometrioma }\end{array}$ & $\begin{array}{l}\text { Tumor Células Ger- } \\
\text { minales Infancia, } \\
\text { Prepubertad) }\end{array}$ \\
\hline REPRODUCTIVA & Quiste Funcional & $\begin{array}{l}\text { EPI } \\
\text { Endometrioma } \\
\text { Quistes paratubarios } \\
\text { Hidrosálpinx } \\
\text { Torsión ovario } \\
\text { Absceso tuboovári- } \\
\text { co }\end{array}$ & $\begin{array}{l}\text { Tumor Epitelial } \\
\text { Fibromas uterinos }\end{array}$ \\
& & $\begin{array}{l}\text { OTROS } \\
\text { Embarazo ectópico }\end{array}$ & \\
\hline POSTMENOPAUSIA & Menos Frecuente & Menos Frecuente & $\begin{array}{l}\text { Tumores Intestinales } \\
\text { Metástasis } \\
\text { Tumor Epitelial }\end{array}$ \\
\hline
\end{tabular}


La evaluación diagnóstica de las mujeres con una masa anexial, incluye la historia familiar y personal de la paciente, el examen físico mediante examen pélvico bimanual, estudios de imagen: ecografía bidimensional y Doppler, Resonancia Magnético Nuclear, Tomografía computarizada; así como pruebas de laboratorio como hemograma, BHCG sérica, marcadores tumorales (CA 125, Inhibinas, deshidrogenasa láctica, Alfa feto Proteína, HE4). Siempre con el fin de descartar malignidad.

El cáncer de Ovario varía en características según su origen celular. Siendo el más común el de origen epitelial $(60 \%)$ seguido del tumor de CélulasGerminales $(28 \%)$ y el de células del estroma (10\%). A su vez los de origen no epitelial, suelen detectarse en estadios más tempranos, presentando mejores tasas de sobrevivencia.

Con respecto al Carcinoma epitelial la mayoría corresponde a tumores benignos o Borderline. Sin embargo el $30 \%$ corresponde a tumores malignos, de estos el $90 \%$ pertenecen al Carcinoma epitelial tipo II, el cual es sumamente agresivo, con rápida diseminación. Por esta razón la mayoría de métodos de diagnóstico se enfocan en la detección específica de este tipo de carcinoma.

El ultrasonido convencional es la modalidad más común para el diagnóstico de masas anexiales. El US transvaginal presenta mejores resultados por su mayor resolución y el US Doppler se utiliza como adyuvante en el diagnóstico, para demostrar aumento de la vascularidad tumoral, así como flujos de baja resistencia.Se han creado Sistemas de calificación o Scores para estandarizar los hallazgos sonográficos y mejorar la detección de malignidad, valorando criterios como consistencia sólida, bordes irregulares, multiecogenicidad, septum irregular, bilateralidad. El Score Sassonees uno de estos y evalúa características de la pared (Grosor y presencia de proyecciones), presencia de tabiques (Grosor), ecogenicidad. Con una sensibilidad de $86 \%$ y especificidad de $77 \%$. $^{(1,4,8,11)}$

Mediante características sonográficas, también se puede hacer sospecha de ciertas entidades benignas, como por ejemplo de un endometrioma o quiste hemorrágico, al presentarse una imagen con múltiples ecos internos, un hidrosálpinx al observarse una dilatación tubular y un teratoma maduro al contener componentes hipoecoicos con múltiples interfaces homogéneas. ${ }^{(1,4,11)}$

CA-125 es un marcador sérico producido por tejidos derivados del epitelio celómico. Presenta baja sensibilidad y especificidad,ya que puede elevarse tanto en condiciones no ginecológicas (gastroenteritis, pancreatitis, hepatitis), así como otras condiciones ginecológicas, (endometriosis, miomatosis, EPI, embarazo) y también existe un $1 \%$ de probabilidad de elevación en pacientes sanas. Una de sus mayores limitaciones, es que solo un $50 \%$ se eleva en carcinoma de ovario de estadío temprano. . $^{(1,4,8)}$

Un nuevo biomarcador recientemente aceptado por la FDA para monitoreo de cáncer de ovario es el HE4(Human EpididymisProtein 4). Corresponde a una glicoproteína expresada solo por células malignas epiteliales del ovario y no se encuentra presente en condiciones benignas. HE4 sería el marcador más sensitivo para cáncer de ovario utilizado hasta ahora. Se demostró adicionalmente que la combinación con CA 125 alcanza sensibilidades aún mayores, que cada uno por separado. ${ }^{(7)}$

Actualmente existen varios scores de predicción de riesgo de malignidad ovárica ROMA (Risk of Ovarian Malignancy Algorithm), RMI, OVA 1, los cuáles permiten clasificar a las pacientes en la categoría de alto o bajo riesgo de detectar cáncer de ovario en la existencia de una masa ovárica. ROMA es un cálculo matemático que utiliza los valores séricos de CA-125 y $\mathrm{HE} 4(\mathrm{U} / \mathrm{ml})$, la edad de la mujeryel estatus menstrual. Mientras el RMI utiliza solamente el CA125. . $^{(1,7,9)}$

El algoritmo ROMA permite estratificar el riesgo de hallar cáncer epitelial de ovario en mujeres que se presentan con una masa pélvica, distinguiéndolo de procesos benignos, permite detectar la enfermedad en estadíos tempranos, y así poder iniciar el tratamiento correspondiente y aumentar la sobrevida del paciente.

Aún se necesita mayor investigación sobre HE4 y ROMA en la diferenciación de masas anexiales, especialmente en mujeres premenopáusicas. Es importante destacar que ninguno de estos se debe realizar como método de screening, ni en sustitución del criterio clínico, debido a que aún se encuentran en proceso de estudios. ${ }^{(7)}$

\section{Tratamiento}

En el manejo de la masa anexial se debe estratificar el riesgo de malignidad, para así determinar el tratamiento.La decisión terapéutica más co- 
rrecta también toma en cuenta hallazgos como riesgo de torsión, ruptura y fertilidad futura. ${ }^{(1)}$ Las masas de bajo riesgo de malignidad, $(<1 \%$ de riesgo) en la mayoría quistes simplesuniloculares, menores de $10 \mathrm{~cm}$, se manejan de acuerdo a la edad de la paciente. ${ }^{(4,8)}$

En mujer premenopáusica se autoriza observación por 3 a 6 meses y se puede indicar tratamiento con ACO con el fin de disminuir los síntomas y recurrencias de quistes. Sin embargo no hay evidencia de presentar regresión de los quistes preexistentes.Si la lesión no desparece o aumenta de tamaño se puede presumir malignidad.Así como el desarrollo de características malignas sonográficas, como tabiques o proyecciones. ${ }^{(1,4,8)}$ Se debe tener en cuenta que mujeres pre y menopáusicas, presentan diferencias en cuanto a la funcionalidad ovárica, por lo que los parámetros de seguimiento son distintos, siendo un volumen máximo aceptado de $5 \mathrm{~cm}$ en pre menopáusicas y $1 \mathrm{~cm}$ en menopáusicas ya es criterio de valoración quirúrgica. ${ }^{(11)}$

El uso del marcador CA-125 en masas de bajo riesgo, se puede usar en pacientes postmenopáusicas, no así en premenopáusicas donde no se encuentra beneficio. Este marcador es de los más utilizados, debido a su amplia investigación.

En mujeres postmenopáusicas con un quiste simple de bajo riesgo se recomienda correlacionar con CA125 u otros marcadores. Si este se encuentra $>35 \mathrm{U}$ por $\mathrm{mL}$, se debe referir a Ginecología Oncológica.Y de lo contrario se puede indicar tomas seriadas del anticuerpo y ultrasonografia. ${ }^{(8,9)}$

Una cirugía prematura para terapia de una masa ovárica funcional no maligna, puede resultar en síndrome adherencial, y afectar fertilidad futura.

En pacientes menopaúsicas se valora el manejo, mediante el uso de CA-125 y factor de riesgo heredofamiliar. El manejo expectante es completamente valido excepto en casos con antecedente heredofamiliar de cáncer de ovario, cáncer de mama, cáncer de colon, cambios en las características del ultrasonido, patología benigna de ovario y aumento del CA-125.

En masas de riesgo intermedio y alto riesgo el tratamiento es quirúrgico. ${ }^{(1)}$

La laparoscopía es una vía segura y de excelencia para masas benignas, con baja tasa de complicaciones en manos de un personal entrenado, para abordar masas anexiales sugerentes de benignidad. Hay una disminución de los días de hospitalización, recuperación rápida y disminu- ción del dolor postoperatorio. Se aconseja cumplimiento estricto con los preoperatorios y seguir adecuadamente los pasos transoperatorios para descartar posible patología maligna. Siendo de un 4-6\% la probabilidad de encontrar malignidades en masas anexiales de aspecto benigno. ${ }^{(5.6)}$ Hay estudios que soportan el uso de vía laparoscópica en cáncer de ovario de estadio temprano, en manos de especialistas en la técnica. ${ }^{(4)}$

La laparotomía es la técnica de excelencia en el manejo de masas con riesgo de malignidad, debido a la necesidad de estadiaje. En múltiples casos se inicia con laparotomía en el abordaje de masas benignas, debido al temor que la lesión corresponda a Cáncer de Ovario. ${ }^{(1)}$

Otra decisión en cuanto al tratamiento es la realización de ooforectomía vs. Quistectomía. La única indicación de ooforectomíabilateral sería mutación BRCA1 y 2 , Endometriosis Grave y elección del paciente. La quistectomía se autoriza en pacientes en su mayoría premenopáusicas, con lesiones benignas. (Cistadenoma, teratoma maduro, endometrioma), ya que es peligroso el derrame de líquido de un quiste de características malignas, con potencial diseminación cancerígena.Actualmente existen novedosas técnicas quirúrgicas para prevención de este derrame de líquido con la técnica de mini laparotomía, no así con laparoscopía. ${ }^{(3,5)}$

La preservación de ovarios vs ooforectomía, se debe evaluar individualmente tomando en cuenta los riesgos y beneficios. ${ }^{(1)}$

\section{CONCLUSIONES}

Debido a la inexistencia de método de screening de cáncer de ovario, un buen manejo y la realización de un adecuado protocolo en los diferentes centros hospitalarios, puede ser una herramienta fundamental en la detección de este tipo de cáncer. Cada centro debería valorar los diferentes métodos de valoración de masas anexiales y sus ventajas y desventajas (costos, especificidad, sensibilidad), scores ultrasonográficos, scores de malignidad y determinar el más adecuado según las posibilidades y necesidades de cada centro. De este modo se disminuye el lapso de tiempo entre la sospecha, detección y referencia a especialista.

Las investigaciones persisten con el fin de descubrir un método eficiente para screening de 
cáncer de ovario, el cual debe cumplir con la característica clave de detección temprana.

\section{BIBLIOGRAFÍA}

1. Liu JH, Zanotti KM. Management of the Adnexal Mass. ObstetGynecol 2011; 117: 1413-1428. 12.

2. Ben- AwiLSmorgick N Tovbin J Fuchs N HalperinRPansky M. Does intraoperative spillage of benign ovarian mucinous cystoadenoma increase its recurrence rate? ObstetGynecol 2010; 202(2): 142.e1-5.

3. Gallo Vallejo JL. Conservación de los ovarios frente a ooforectomía bilateral en pacientes sometidas a histerectomía por procesos benignos. ClinInvest Gin Obstet 2009; 36(3): 94-98.

4. Nezhat C Cho J King LP Hajhosseini B Nezhat F. Laparoscopic management of adnexal masses. ObstetGynecolClin North Am. 2011; 38(4): 663-676.

5. Canis M Abischong B Houlle C Botchorishvili R Jardon K Safi A, et al. Laparoscopic management of adnexal masses: a gold standard? CurrOpinObstetGynecol 2002; 14: 423-428.

6. Alejandra catalanB Domingo LaizRJaimeCorvalanDiegomeosliWladi mirVasquez. Manejo laparoscópico de masa anexiales. Revista Chilena de Obstetricia y Ginecologia 200570(4): 231-235

7. Moore Richard G Miller M Disilvestro P Landrum L Gajewski, W Ball J et al. Evaluation of the Diagnostic Accuracy of the Risk of Ovarian Malignancy Algorithm in Women With a Pelvic Mass, Obstetrics \&Gynecology:August 2011 Volume 118 - Issue 2, Part 1 - pp 280288,

8. Givens VMitchelGHarraway-smith C Reddy A David 1. Diagnosis and Managementof Adnexal Masses. 2009 American Academy of FamilyPhysicians, October 15, 2009 Volume 80, Number 8: P815-820.

9. McDonald JM1 Doran S DeSimone CP UelandFRDePriest PD Ware RA et
al.Predicting risk of malignancy in adnexal masses.Obstet Gynecol. 2010 Apr;115(4):687-94.

10. Van den Akker PA1 Aalders AL Snijders MP Kluivers KB Samlal RA Vollebergh JH Massuger LF. Evaluation of the Risk of Malignancy Index in daily clinical management of adnexal masses. GynecolOncol. 2010 Mar;116(3):384-8.

11. Zavaleta-Landa JM Sanchez,JGutierrez.DLMartines.D.

Característicasultrasonograficas benignas y malignas de masas anexiales.RevInvMed Sur Mex, Abril-Junio 2013; 20(2): 130-132

\section{CONFLICTOS DE INTERES}

Los autores declaran que no existe ningún conflicto de interés. 